\title{
A Survey on Integrated Wireless Network Architectures
}

\author{
Khalid. H. Mohammadani ${ }^{[1]}$ \\ Isra University Hyd, Pakistan \\ khalid.mohammadani@gmail.com \\ Ibraheem Channa ${ }^{[3]}$ \\ Quest University, Nawabshah, Pakistan \\ Ibrahim.channa@quest.edu.pk
}

\author{
Hameedullah Kazi ${ }^{[2]}$ \\ Isra University Hyd, Pakistan \\ hkazi@isra.edu.pk \\ Danish Vasan ${ }^{[4]}$ \\ Isra University Hyd, Pakistan \\ touheed_wassan007@yahoo.com
}

\begin{abstract}
Operation and maintenance of installed wireless networks face various difficulties due to rapidly shifting trends in technology. The planning of existing wireless networks make it difficult to combine different elements, made by different technological companies and different network operators. In this paper, we survey different integrated wireless networking structure, system architectures and its applications. Integrated wireless networks use combination of different types of following networks e.g. MANET with mobile cellular network, MANET with satellite network, and GSM with software-defined radio (DSR) that have been proposed for low cost of network deployments and provides the voice communication, short message service and low rate data service for remote areas where infrastructure networks are difficult to exist. They also improve their routing reliability and maintain security issues.
\end{abstract}

\section{Keywords}

Integrated Wireless networks; MANET; Multi-hop network; cellular network

\section{INTRODUCTION}

For last 25 years there has been continue growth in mobile communication due to personal mobility in communication and subscriber demand. In recent study, there are more than 4.0 billion population of world has become mobile users that is almost half of the world population by year 2011 [1,2]. There are so many mobile devices are available in market that are enabled for integrated communication with functionality of FM radio, video player, sound player, Bluetooth and GPRS capabilities etc. New services need high bandwidth and quality of services.

Currently there are two main limitations capacity and the presence of unbalanced traffic in any mobile cellular network[3], therefore it faces so many problems due to rising demand of subscribers.

In this paper we survey integrated cellular technology. The reminder of this survey paper is organized as follows. Section 2 concept of Infrastructure and infrastructure less, section 3 proposed wireless integrated networks section 4 compressions of architectures at the end section 5 concludes the paper.

\section{INFRASTRUCTURE NETWORK AND INFRASTRUCTURE-LESS NETWORK}

Mobile station exchanges information directly with BTS in wireless infrastructure network which known as single-hop cellular system [4]. MS can move during the call and base stations transceivers (BTS) are fixed and when MS goes out of one BTS's range then it hands over the range of another BTS[5] as in Fig.1. The cellular system and conventional system are best examples of infrastructure network.

In infrastructure-less network is temporary and self organizing network. The concept of ad hoc network is not new, have been approximately in different structure for over last 20 years. A infrastructure-less network which is mostly identified by mobile ad-hoc network (MANET) in which no any concept of fixed BS or access point (AP) node connected to MS/PC. Every node works as router and node also can move easily while communicating. They establish dynamic routing among the nodes to develop their own network [5]. Path is established from source to destination node by node for the communication as in fig 2 .

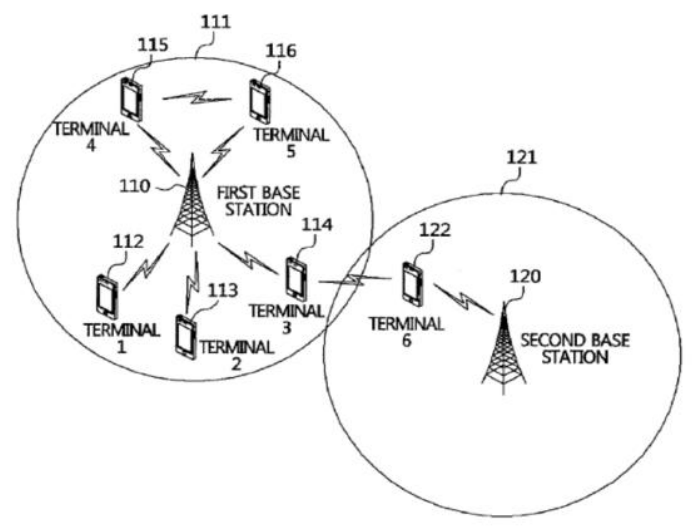

Fig 1: Infrastructure network[6] 
Ad-hoc network can be integrated with fixed existing technologies like cellular that is not part of ad-hoc structure. The performance and service of fixed network are improved by using the ad-hoc network. And ad-hoc networks can give connections among the subscribers where fixed infrastructure is not available [8].

\section{PROPOSED INTGRATED WIRELESS NETWORK ARCHITECTURES}

There were many integrated wireless networks are designed for various purposes in the previous a few years. Some of them are proposed for dedicated gateways, relaying and access points for traffic forwarding. These devices may be fixed elements of some networks.

\subsection{Integrated Maritime Communication System}

The architecture proposed in [9], namely Integrated maritime communication system (IMCS) merged with three various wireless systems i.e. Vehicular ad-hoc network among the ships, cellular networks \& satellite mobile network. Vehicular ad-hoc network is known as VANET which is a type of MANET in which vehicular acts as a node and communicates with each another [10], most of research is being focused on road traveling for carriages [11]. Authors also discussed that possible communication to create VANET wireless mesh topology among the boats at sea. The advantage of VANET among the ships is that they could communicate with each another without satellite at sea which is for from shore area. For ship to shore communication they suggested VANET and cellular mobile network when they are within range of base station that is fixed at shore area and seaport. They suggested ship borne cellular gateway (C-GW) on one ship to finish all mobiles directly access with base station. When the ships are not in the range of cellular mobile network only for that situation they involved in satellite network for shore to ship communication. When they want to get access satellite network first they access VANET then connect to satellite mobile network via a ship borne satellite gateway that is on one ship. Cellular network connects with satellite network using a terrestrial cellular gateway on earth. In fig. 3 Architecture of IMCS is showed.

Authors also proposed that, the function of integrated maritime services system (IMSS) is adding together with IMCS for giving value added services such as locations, voice communication, fishery reports and entertainment. The main focus of system assists to decrease the network operation cost and call charges, supports more workstation, and provides comfortable maritime services to mobile users. The scenarios of using satellite system are reduced, which yields the advantage of decreased calling charge.

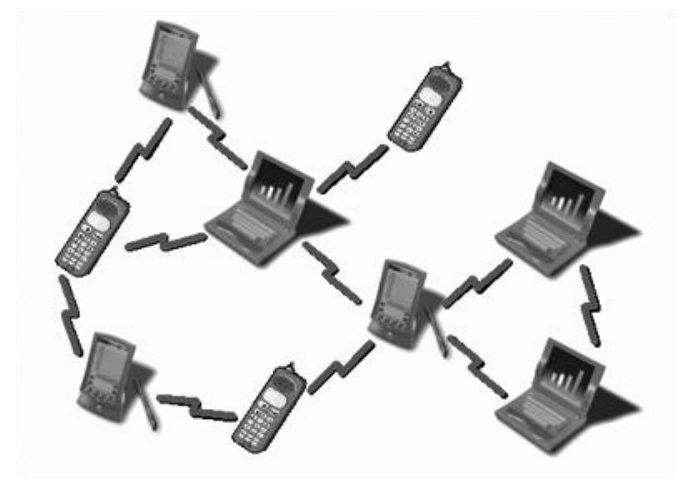

Fig 2: Infrastructure-less network[7]

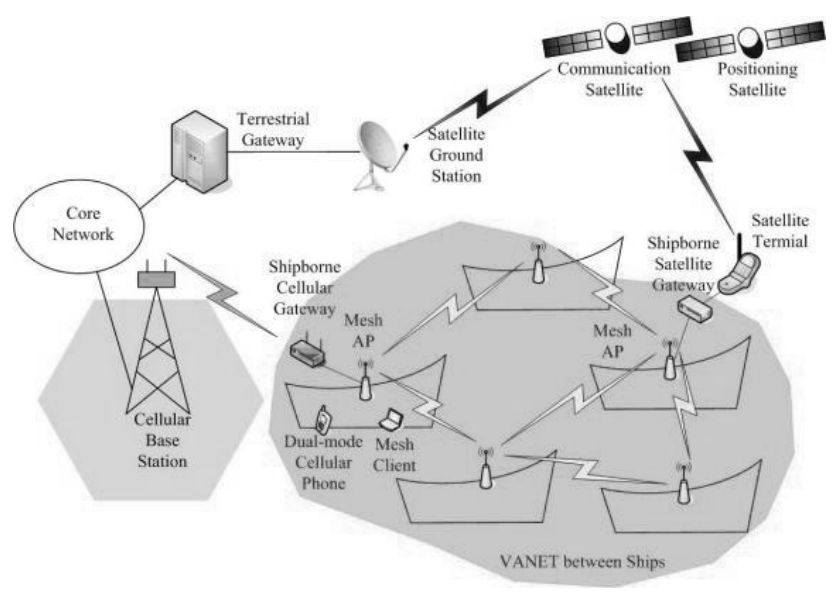

Fig 3: Architecture of IMCS[9]

\subsection{A Wireless Architecture Using MANET For Mobile Communications}

A wireless networking architecture using MANET for mobile communications of remote pastoral areas in Tibet was proposed in [12], which may decreases the system operation cost. Mobile subscribers helped by the solar powered standing station focus on network maintenance and routing deal in MANET. The mobile ad-hoc users connect to cellular network and internet via MANET gateway whose coverage range is more than common BTS as showed in fig.4. Only authorized devices contact to MANET gateway because it is authorized hub. They suggested two types of nodes, one is standing and second is mobile node but their work is different in tradition self-organized network. The standing station is supported by solar system when solar power is more powerful in summer at Tibet. It helps to connect mobile users with cellular gateway when there is no any network signal of own cellular network at mobile station. Each gateway connects more than one standing stations. Some time mobile devices have accessing of Beidou message accessing to select for different situations which support maximum sharing. In suggested system, mobile nodes have been suggested as multiple accessing modes for connecting with cellular network, MANET and Beidou satellite. In last they suggested a design of cross layer to improve network efficiency and save energy. 


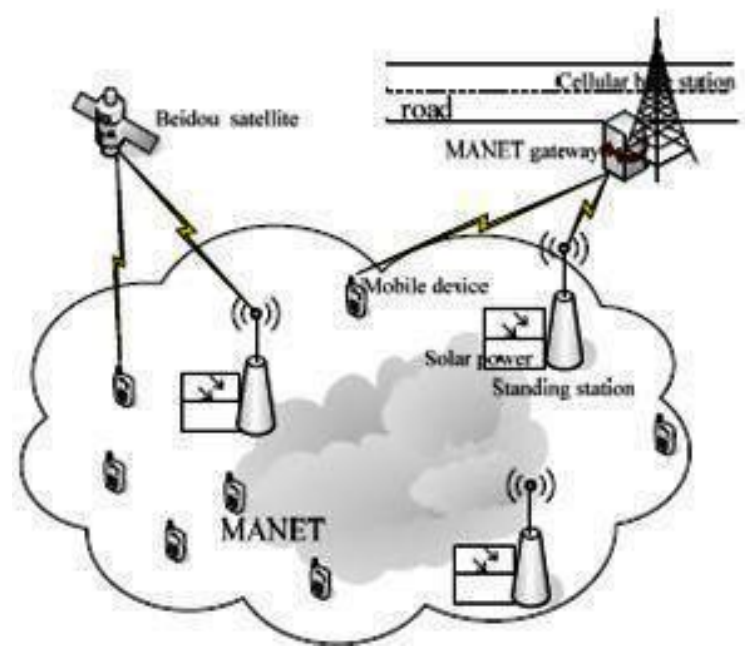

Fig 4: Wireless networking architecture using MANET[12]

\subsection{A Cognitive Mobile BTS Solution With SDR Electric Sensing}

In [13] this design, authors suggested software-define radio (SDR) that can give permission of low-price deploying and possibly, value added services are not in traditional cellular network. They suggested ships as vehicles using vehicular adhoc network (VANET), showed in fig.5.

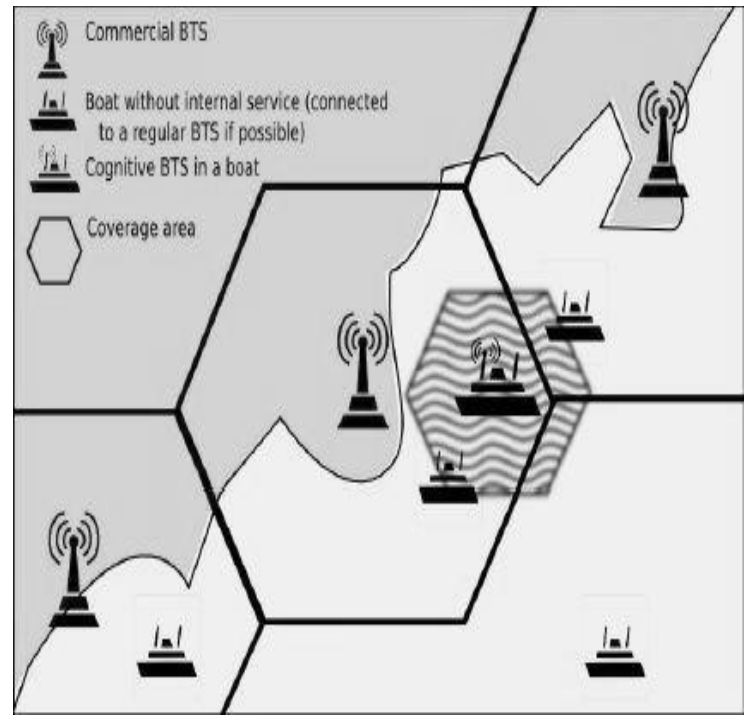

Fig 5: Architecture of Cognitive mobile BTS System overview [13]

They used a mobile cognitive BTS that takes benefit from flexible SDR for value-added services in emergency situation because it has abilities of shared spectrum. Authors proposed low-price BTS for cognitive mobile technology. In this, they communicated with traditional BTS and in addition it provides cognitive sensing attributes to avoid interfaces with secondary base station transceiver and other vehicles, during the communication services indoors the vehicles for real-time applications. They refined and checked more serious parts of system and representing the right performance and validity.

\subsection{An Integrated Cellular And Ad-hoc Relaying System}

An architecture suggested in [14], integrated cellular and ad-hoc relaying (iCAR), a typical proposal for multi-hop communication networks through fixed relays that makes usage of standard cellular technology with ad-hoc networking technology to recognize the dynamic load balancing.

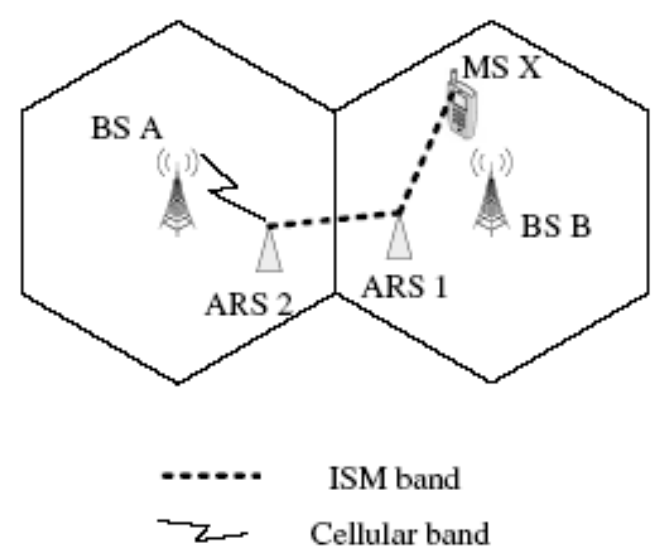

Fig 6: The primary relaying strategy in iCAR[3]

The main concept of iCAR is to strategically search the no. of fixed relays, known ARSs (ad-hoc relay stations), and apply them to redirect traffic from individual possible congested cell to additional uncongested cells. Therefore, the congestion is being able to be eliminated. After that, iCAR system creates this achievable to manage handover voice calls for mobile stations moving into a heavily traffic cell or to attend new calls request generated from mobile stations in a densely cell. when Mobile station $\mathrm{X}$ does not find a cellular channel frequency in cell $\mathrm{B}$ to set up a communication link with Base station $\mathrm{B}$, it would send the data to its nearest ARS, ARS 1, ISM band that is not in range of cellular band. As shown in figure.6, ARS 1 sends data, using ISM band next to another ARS known as ARS 2 that is within the range of neighbor cell that is cell A. At last, ARS 2 would forward the data to base station A by using cellular frequency channel. It gives valuable technique for the heavy traffic problem with dynamically balancing the data load among different geographical cell areas. By the load balancing, iCAR can extend the coverage range of typical single hop communication networks. it is right because if mobile station is away from coverage range of the base station that can access the system with help of ARS. The plan on deploying ARSs is examined in $[14,15]$ that studied how to create a range-free topology designed for ARSs consequently that scalability can be gained. By means of the range-free topology of ARSs, they suggested a load-balancing-based routing method for iCAR thus the system is additional robust to base station breakdowns and the present resources can be used powerfully.

Moreover, the performance finding of iCAR is presented in [16]. The analysis is performed by a 2-d Markov chain model based on the finite number of orthogonal channels for cellular band and limited numbers of orthogonal channels for industrial, scientific and medical(ISM) radio bands. 


\subsection{Cellular-aided Ad hoc Network}

The architecture proposed in [17], namely a cellular-aided ad hoc network (CAMA). It is another kind of integrating heterogeneous wireless system that is used in that places where a mobile ad hoc network overlaps a cellular network where traffic relayed by mobile stations. CAMA agents are operated by servers BS. Each and every CAMA agent is linked with server base stations to manage the control information connected to multi-hop transmission. Mobile stations may contact to CAMA agent for exchanging the routing path and security information through the cellular radio channel by help of BSs.

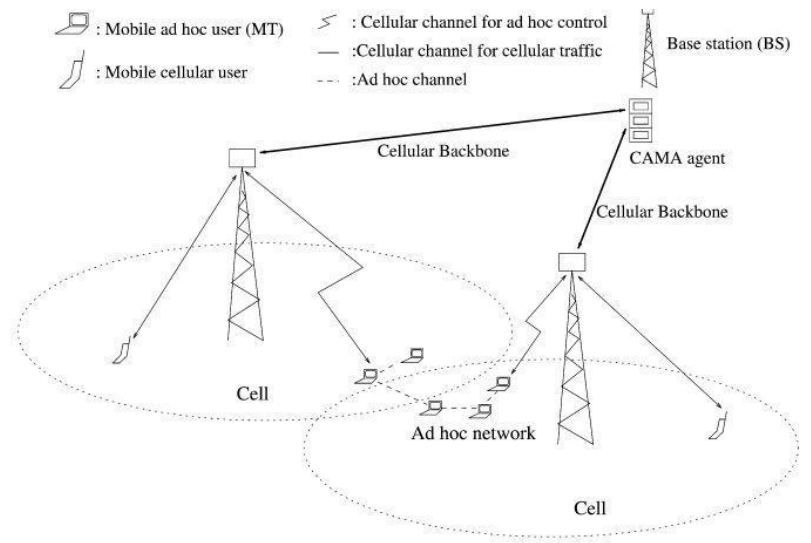

Fig 7: cellular-aided mobile ad hoc network [17]

Furthermore, MS is being able to path discovery to recognize the mobile relay stations with help of CAMA agents because all mobile stations are assumed to have global positioning system (GPS) capabilities. So a CAMA agent function is used as a location information server. The authors in [17] declared that low charge high data rate ad hoc channels are right for multimedia services and utilize it thought as drive to forward all traffic transfer by the mobile ad hoc network. CAMA is not a general architecture for various wireless networks however it uses the cellular infrastructure to get better the performance of mobile ad hoc networks [18]. CAMA is recommended in [19] that are stronger than pure ad hoc network.

\section{COMPARISIONS OF ARCHITECURES}

We have selected a few criteria for comparison of integrated wireless network architectures and multi-hop communication network architectures: Network cost, network planning, adjusting to network development, routing protocol, quality of services and power supply.

\subsection{Network cost}

By Using MANET, network cost will be decreased as well as deployment cost although more cheap than fixed BS. Mobile devices may be a little bit costly because they use two modes and two antennas to work as router, received data from one node and forward it to other node and it is also for avoiding the selfinterface. Also, the Mobile station needs a strong computational element to manage the complex routing algorithm[1].

\subsection{Network planning}

When fixed base station is used on the sites of network performance and the no. of fixed base stations by considering the exchange between networks strategies should be determined
[14]. However, mobile relays are used in integrated wireless network without any problem.

\subsection{Adjusting to network development}

When mobile operators use base station with fixed relays, it may be costly and difficult for re-located but mobile stations are used as relays for adopting network growth without such difficulties.

\subsection{Routing protocol}

With fixed AP/relay it is very easy rather than MS relay because MS knows very well for fixed AP/relay to help routing decision for next updating of path and fixed relays have also fewer difficulties to handle mobility of Mobile subscribers than mobile station relay[15].

\subsection{Quality of Service (QoS)}

Cellular with fixed access point or fixed relay gives good quality of services [15], reduces latency and increases throughput. MANET's protocol has ability to overcome the rout problem due to efficient protocol there is very less disturbing in the traffic arriving at destination.

\subsection{Power supply}

There is some issues of power for mobile stations but no power consumption issue for fixed AP/relay[20] at ships or standing stations.

\section{CONCLUSIONS}

Maintenances of large network are difficult in emergencies situations especially at sea areas or those areas which are very far from the urban area. Wireless technology has been quickly growing. Integrated wireless architectures assure to use benefits of common wireless cellular network and rising ad-hoc relay systems to improve the overall system throughout their routing capability and to maintain security issues.

In this paper, we visited different selected concept of integrated wireless network joint with heterogeneous networks which provide large distances communication, value added services, multimedia services for poor network communication. And we compared them for some criteria and discussed their respective technical strength and issues.

Integrated wireless technology is hence considered to able $4 \mathrm{G}$ and 5G network applicant for future cellular network. Though, it needs suitable system architecture before all these benefits will be able to be realized. The usage of mobile relay systems in network development can adapt dynamically because it will raise the complexity of designing and routing protocols for mobile stations. Researcher can do more research on better performance of routing protocols and MAC for dynamic mobile relay model. Wireless network operators should consider an exchange between the network implementation complexity and the system performances of integrated wireless networks.

Table. 1 shows different existing integrated wireless network that are suggested for different objectives using different ways of wireless technology. 
Table 1. Comparison of integrated wireless architectures

\begin{tabular}{|c|c|c|c|}
\hline Architectures & $\begin{array}{l}\text { Wireless } \\
\text { technologie } \\
\text { s }\end{array}$ & Interfaces & Main optimization goal \& implementation \\
\hline IMCS & $\begin{array}{l}\text { Cellular, } \\
\text { WLAN, } \\
\text { Satellite, } \\
\text { VANET }\end{array}$ & $\begin{array}{l}\text { Single \& } \\
\text { Dual }\end{array}$ & $\begin{array}{l}\text { Solving the interface problem because of overcrowding on the } \\
\text { waveband and atmospheric disturbance. } \\
\text { Used VANET devices with satellite and cellular network for } \\
\text { traffic relaying. }\end{array}$ \\
\hline $\begin{array}{l}\text { MANET for remote } \\
\text { areas in Tibet }\end{array}$ & $\begin{array}{l}\text { Cellular, } \\
\text { WLAN }\end{array}$ & Dual & $\begin{array}{c}\text { Solving the network deployment and maintenance cost. } \\
\text { Provides communication at rural areas of Tibet. }\end{array}$ \\
\hline SDR radio & $\begin{array}{l}\text { Conventiona } \\
1 \text { cellular }\end{array}$ & Single & $\begin{array}{l}\text { A mobile cognitive BTS through spectrum sensing capabilities to } \\
\text { take help of SDR technology. } \\
\text { Cognitive BTS gives communication among the vehicles and } \\
\text { avoid interference in real communication. }\end{array}$ \\
\hline iCAR & $\begin{array}{l}\text { Cellular, } \\
\text { WLAN, } \\
\text { MANET }\end{array}$ & $\begin{array}{l}\text { Single \& } \\
\text { Dual }\end{array}$ & $\begin{array}{l}\text { Solving the congestion problem to improve load balance between } \\
\text { BSs. } \\
\text { For the network traffic strategically placing needs fixed relaying } \\
\text { devices i.e ARS }\end{array}$ \\
\hline CAMA & $\begin{array}{l}\text { Cellular } \\
\text { MANET }\end{array}$ & $\begin{array}{l}\text { Single \& } \\
\text { Dual }\end{array}$ & $\begin{array}{l}\text { To overlaid on ad hoc network. } \\
\text { Only using CAMA agents for getting to access to gateways. }\end{array}$ \\
\hline
\end{tabular}

\section{REFERENCES}

[1] Li, Xue Jun, Boon-Chong Seet, and Peter Han Joo Chong. "Multihop cellular networks: Technology and economics." Computer Networks 52.9 (2008): 1825-1837.

[2] The Portio Research Limited, Worldwide Mobile Market Forecasts 2006-2011. Market Study, UK, 2006.

[3] $\mathrm{Wu}$, Hongyi, et al. "Integrated cellular and ad hoc relaying systems: iCAR." Selected Areas in Communications, IEEE Journal on 19.10 (2001): 2105-2115.

[4] C.N. Thurwachter Jr., Wireless Networking, Prentice Hall, New Jersey, US, 2002.

[5] Taneja, S., \& Kush, A. (2010). A Survey of routing protocols in mobile ad hoc networks. International Journal of Innovation, Management and Technology, 1(3), 2010-0248.

[6] Ahn, Jae Young, Choong Il Yeh, and Kyoung Seok Lee. "METHOD FOR DEVICE-TO-DEVICE COMMUNICATION BASED ON CELLULAR TELECOMMUNICATION SYSTEM." U.S. Patent No. 20,130,083,779. 4 Apr. 2013.

[7]http://www.google.com.pk/search?q=infrastructure+less+net work $\&$ biw $=1280 \&$ bih $=843 \&$ source $=1 \mathrm{nms} \& \mathrm{tbm}=\mathrm{isch} \& \mathrm{sa}=$ X\&ei=z6D7UcutEqvc4QS044FQ\&ved=0CAcQ_AUoAQ
[8] Minhas, Qurratul-Ain, Hasan Mahmood, and Hafiz Malik. "The role of ad hoc networks in mobile telecommunication." RECENT DEVELOPMENTS IN MOBILE COMMUNICATIONS-A MULTIDISCIPLINARY APPROACH (2011): 179.

[9] Du, Wencai, et al. "Integrated Wireless Networking Architecture for Maritime Communications." Software Engineering Artificial Intelligence Networking and Parallel/Distributed Computing (SNPD), 2010 11th ACIS International Conference on. IEEE, 2010.

[10] Arulkumar, N., and E. Raj. "A simulation based study to implement Intelligent Transport Systems concepts in VANETs using AODV routing protocol in NS2." Advanced Computing (ICoAC), 2012 Fourth International Conference on. IEEE, 2012.

[11] J.J. Blum, A. Eskandarian, and L.J. Hoffman, "Challenges of Intervehicle Ad Hoc Networks," IEEE Trans. on ITS, Vol.5, No.4 2004, pp. 347-351

[12] Huang, Cao, Xiaojun Guo, and Zeguo Liu. "A wireless networking architecture using MANET for mobile communications of remote pastoral areas in Tibet," ICCSEE 2013. 
[13] Muñoz, Jorge, et al. "A Cognitive Mobile BTS Solution with Software-Defined Radioelectric Sensing." Sensors 13.2 (2013): 2051-2075.

[14] S. Dixit, E. Yanmaz, O.K. Tonguz, On the design of selforganized cellular wireless networks, IEEE Communications Magazine 43 (7) (2005) 86-93.

[15] SHARMA, V., \& JAIN, D. (2010). Multihop cellular networks: a review. International Journal of Engineering Science, 2(11), 6082-6091.

[16] E.Yanmaz, O.K. Tonguz, Dynamic load balancing and sharing performance of integrated wireless networks, IEEE Journal on Selected Areas in Communications 22 (5) (2004) 862-872.

[17] B. Bhargava, X. Wu, Y. Lu, W. Wang, Integrating heterogeneous wireless technologies: a cellular aided mobile ad hoc network (CAMA), Mobile Networks and Applications 9 (2004) 393-408.

[18] Cavalcanti, Dave Alberto Tavares. Integrated architecture and routing protocols for heterogeneous wireless networks. Diss. University of Cincinnati, 2006.

[19] Bhargava, Bharat, et al. "Integrating heterogeneous wireless technologies: a cellular aided mobile ad hoc network (CAMA)." Mobile Networks and Applications 9.4 (2004): 393-408

[20]N.B. Salem, L. Buttyan, J.-P. Hubaux, M. Jakobsson, A charging and rewarding scheme for packet forwarding in multi-hop cellular networks, in: Proceedings of ACM MobiHoc'03, Annapolis, Maryland, USA, 2003, pp. 13-24. 